\title{
The relationship between wormlike micelle scission free energy and micellar composition: The case of Sodium Laurylethersulphate and Cocamidopropyl Betaine
} DOI:

10.1021/acs.langmuir.0c02210

\section{Document Version \\ Accepted author manuscript}

Link to publication record in Manchester Research Explorer

Citation for published version (APA):

Wand, C., Carbone, P., Panoukidou, M., Del Regno, A., \& Anderson, R. (2020). The relationship between wormlike micelle scission free energy and micellar composition: The case of Sodium Laurylethersulphate and

Cocamidopropyl Betaine. Langmuir. https://doi.org/10.1021/acs.langmuir.0c02210

\section{Published in:}

Langmuir

\section{Citing this paper}

Please note that where the full-text provided on Manchester Research Explorer is the Author Accepted Manuscript or Proof version this may differ from the final Published version. If citing, it is advised that you check and use the publisher's definitive version.

\section{General rights}

Copyright and moral rights for the publications made accessible in the Research Explorer are retained by the authors and/or other copyright owners and it is a condition of accessing publications that users recognise and abide by the legal requirements associated with these rights.

\section{Takedown policy}

If you believe that this document breaches copyright please refer to the University of Manchester's Takedown Procedures [http://man.ac.uk/04Y6Bo] or contact uml.scholarlycommunications@manchester.ac.uk providing relevant details, so we can investigate your claim.

\section{OPEN ACCESS}




\title{
The relationship between wormlike micelle
}

\section{scission free energy and micellar composition:}

\section{The case of Sodium Laurylethersulphate and}

\section{Cocamidopropyl Betaine}

Charlie R. Wand, ${ }^{*, \dagger}$ Maria Panoukidou, ${ }^{\dagger}$ Annalaura Del Regno, ${ }^{\ddagger} \uparrow$ Richard L. Anderson, ${ }^{\ddagger}$ and Paola Carbone ${ }^{\dagger}$

$\dagger$ Department of Chemical Engineering and Analytical Science, University of Manchester, Oxford Road, Manchester, M13 9PL, United Kingdom

$\ddagger S T F C$ Hartree Centre, Scitech Daresbury, Warrington WA4 4AD, United Kingdom ๑BASF SE, Materials Molecular Modeling, Carl Bosch Str. 38, 67056, Ludwigshafen, Germany

E-mail: Charlie.wand@manchester.ac.uk

\begin{abstract}
The scission energy is the difference in free energy between two hemispherical caps and the cylindrical region of a wormlike micelle. This energy difference determines the logarithm of the average micelle length, which affects several macroscopic properties such as the viscosity of viscoelastic fluids. Here we use a recently published method by Wang et al (Langmuir 201834 1564-1573) to directly calculate the scission energy of micelles composed of monodisperse sodium laurylethersulphate ( $\left.\mathrm{SLES}_{\mathrm{n}} \mathrm{EO}\right)$, an anionic surfactant. Utilising dissipative particle dynamics (DPD), we perform a systematic study varying the number of ethoxyl groups $(n)$ and salt concentration. The scission
\end{abstract}


energy increases with increasing salt concentration, indicating that the formation of longer micelles is favoured. We attribute this to the increased charge screening that reduces the repulsion between head groups. However, the scission energy decreases with increasing number of ethoxyl groups as the flexibility of the head group increases and the sodium ion becomes less tightly bound to the head group. We then extend the analysis to look at the effect of a common co-surfactant, cocamidopropyl betaine (CAPB) and find that its addition stabilises wormlike micelles at a lower salt concentration.

\section{Introduction}

Surfactants are molecules comprised of a hydrophobic tail covalently bonded to a hydrophilic head group. These competing interactions lead surfactant systems to self-assemble into a wide range of microstructures in aqueous solution, ranging from small spherical micelles to lamellar structures. The resulting microstructure is dependent on the temperature, pressure and surfactant concentration, ${ }^{1,2}$ making the mapping of phase diagrams non-trivial. In addition, for ionic systems the presence of salt can drastically change the microstructure and the viscous properties of the fluid. For example, at a fixed surfactant concentration, the viscosity of anionic surfactant solutions such as sodium laurylethersulphate (SLES) considered here can vary by over twenty times. ${ }^{3}$ This rheological behaviour has been related to the change in morphology of the micelles. It has been suggested that at low salt concentrations spherical micelles are observed, with a similar zero shear viscosity as pure water. As the salt concentration increases, the micelles begin to grow in length, causing the viscosity to increase. There is a peak in the zero shear viscosity at intermediate salt concentrations due to long, entangled wormlike micelles. As the salt level continues to increase, the viscosity decreases again as branches begin to form, allowing for a additional stress relaxation mechanisms including branch sliding and phantom crossing. ${ }^{4-6}$ Due to this rich phase behaviour, surfactant systems have found application in the oil/gas industry ${ }^{7}$, as detergents $^{8}$ and in nature in the guise of cell membranes. ${ }^{9}$ Here we concentrate on one such microstructure, wormlike micelles 
(WLM). These micelles can be up to microns in length, whilst maintaining a diameter on the nanoscale and bear many common properties with polymers, with the added ability to break and reform, earning the name "living polymers". ${ }^{10}$ WLM are an industrially important phase with many personal care products exhibiting this microstructure. However, the isotropic nature and comparatively large size with respect to smaller spherical micelles, means that the WLM microstructure is less well characterized than other phases. Common experimental techniques employed to categorise anisotropic liquid crystalline phases (e.g. polarized optical microscopy) or smaller aggregates such as spherical micelles ( e.g. dynamic light scattering) can not be applied to these systems. However, recent experimental approaches employing small angle neutron scattering (SANS) by $\operatorname{Vogtt}^{11,12}$ on dilute solutions and the development of an iterative mesoscopic model to extract the information from rheological data by Zou and Larson ${ }^{13}$ has made progress in this area. In addition, several theoretical approaches have been developed exploiting the similarity of WLM to conventional polymers. ${ }^{14-16}$ Cates and co-workers ${ }^{14}$ borrow from the reptation theory of entangled polymer to allow for quantitative predictions of physical properties such as aggregation number and mean micelle length, $\bar{L}$, given by

$$
\bar{L}=\Psi^{y} \exp \left(\frac{E_{s c i}}{2 k_{B} T}\right)
$$

where $k_{B}$ and $T$ are the Boltzmann constant and temperature whilst $\Psi$ is the micelle volume fraction and $y$ is an exponent that is usually taken to be $\frac{1}{2}$ for a system with Gaussian statistics. ${ }^{17,18} E_{s c i}$ is the scission energy, defined as the difference in free energy between two hemispherical caps and the cylindrical body of a WLM. As with conventional polymeric solutions, $\bar{L}$ and thus $E_{\text {sci }}$ plays a defining role in the zero-shear viscosity. Despite the importance of $E_{s c i}$ its value cannot be measured directly but rather it relies on the measurement of micelle size/length and the use of equation $1 .{ }^{19}$

Theoretical approaches have also been employed to calculate the $E_{\text {sci }}$, these build on the geometric approach by Israelachivili ${ }^{20,21}$ and Tanford ${ }^{22}$ and the molecular thermodynamic 
approach of Nagarajan. ${ }^{23,24}$ Recently Danov et al predict the scission energy for both single component ${ }^{25}$ and multiple component ${ }^{26}$ non-ionic WLM finding good agreement with experimental results. However, as is common in theoretical approaches, several approximations about the nature of the surfactant molecules are made.

Computer simulations offer the ability to investigate the properties of a micelle at a molecular level. Bulk system self-assembly simulations where the micelles are allowed to form spontaneously from a randomly dispersed mixture can provide information on the size distribution. When used conjunction with equation 1 , this is a conceptually simple approach to calculate $E_{s c i}$. However, this method requires extremely large system sizes to be statistically accurate, particularly in the region where WLM are formed as these aggregates can be on the order of microns in length which is not currently accessible to molecular level simulation techniques such as molecular dynamics. An alternative method was recently proposed by Wang et $a l^{27}$ using umbrella sampling to directly calculate the change in free energy upon scission, thus avoiding the finite size effects of the bulk system simulations. Here we employ the method proposed by Wang et al to investigate WLM composed of monodisperse SLES. SLES is an anionic surfactant with the chemical formula $\mathrm{CH}_{3}\left(\mathrm{CH}_{2}\right)_{\mathrm{m}}\left(\mathrm{OCH}_{2} \mathrm{CH}_{2}\right)_{\mathrm{n}} \mathrm{OSO}_{3}{ }^{-} \mathrm{Na}^{+}$(Fig 1$)$ and the base of many personal care products where $m=11$ is the dominant species, however in industrial applications a distribution of both $m$ and $n$ are found. Here we perform a systematic study in the salt- $n$ composition space, where $n$ is the number of $\mathrm{OCH}_{2} \mathrm{CH}_{2}$ (EO) groups. Literature on pure, monodisperse SLES is sparse and data is more commonly available as a mixture of tail length $(m)$ and EO number $(n) .{ }^{28-30}$ By performing simulations on monodisperse SLES, we can elucidate the fundamental effects of the salt concentration and number of EO groups on $E_{s c i}$. We then go on to investigate the addition of cocamidopropyl betaine (CAPB) on $E_{s c i}$. CAPB is a zwitterionic surfactant with the formula $\mathrm{CH}_{3}\left(\mathrm{CH}_{2}\right)_{\mathrm{m}}(\mathrm{CO}) \mathrm{NH}\left(\mathrm{CH}_{2}\right)_{3} \mathrm{~N}^{+}\left(\mathrm{CH}_{3}\right)_{2} \mathrm{CH}_{2} \mathrm{COO}^{-}$ that is often added to SLES as a thickener, antistatic agent and to reduce skin irritation. As in the case of SLES, for CAPB $m=11$ is the dominant species with a distribution of $m$ 
found in industrial applications.

Due to the micron length scales involved in these WLM systems we employ a mesoscopic simulation approach, dissipative particle dynamics (DPD) in which groups of atoms are represented by soft repulsive beads. ${ }^{31}$ DPD has previously been extensively used to study polymer ${ }^{32,33}$ and surfactant solutions, ${ }^{34}$ including the calculation of phase diagrams and critical micelle concentrations. ${ }^{35,36}$

The paper is organised as follows. Firstly we present the model and methods used, before presenting and discussing the results. We separately discuss the affect of salt concentration and $n$ on pure, monodisperse SLES micelles before considering the mixed micellar systems. We then summarise our findings and discuss future directions.

\section{Experimental}

\section{Model}

We employ DPD, a coarse-grained approach in which groups of atoms are modelled as soft, purely repulsive beads which allows for a relatively large time step in comparison to conventional molecular dynamics simulations. ${ }^{31}$ In DPD, the force acting on particle $i$ is given by

$$
\boldsymbol{F}_{i}=\Sigma_{j \neq i}\left(\boldsymbol{F}_{i j}^{D}+\boldsymbol{F}_{i j}^{R}+\boldsymbol{F}_{i j}^{C}+\boldsymbol{F}_{i j}^{B}+\boldsymbol{F}_{i j}^{c o u l}\right)
$$

where $\boldsymbol{F}_{i j}^{D}, \boldsymbol{F}_{i j}^{R}, \boldsymbol{F}_{i j}^{C}$ are the dissipative, random and conservative forces respectively on particle $i$ from $j$. The final two terms correspond to bonded and coulombic interactions respectively.

The dissipative and random forces are given by

$$
\begin{aligned}
& \boldsymbol{F}_{i j}^{D}=-\gamma \omega^{D}\left(\boldsymbol{r}_{i j}\right)\left(\hat{\boldsymbol{r}}_{i j} \cdot \boldsymbol{v}_{i j}\right) \hat{\boldsymbol{r}}_{i j} \\
& \boldsymbol{F}_{i j}^{R}=\Delta t^{-1 / 2} \sigma \omega^{R}\left(r_{i j}\right) \xi_{i j} \hat{\boldsymbol{r}}_{i j}
\end{aligned}
$$


where $\boldsymbol{r}_{i j}$ is the inter-particle distance between beads $i$ and $j, \boldsymbol{v}_{i j}$ is their relative velocity and $\Delta t$ is the time step. $\omega^{D}\left(r_{i j}\right)$ and $\omega^{R}\left(r_{i j}\right)$ are distance-dependent weighting functions and $\xi_{i j}$ is a Gaussian-distributed random variable with zero mean and unit variance where $\xi_{i j}=\xi_{j i}$. Note the hat stands for a unit vector, i.e. $\hat{\boldsymbol{r}}_{i j}=\boldsymbol{r}_{i j} / r_{i j}$. To ensure that momentum is conserved, $\boldsymbol{F}_{i j}^{D}$ and $\boldsymbol{F}_{i j}^{D}$ are related ${ }^{37}$ with the friction coefficient, $\gamma$, related to the random force strength, $\sigma$, by

$$
\frac{\sigma^{2}}{k_{B} T}=2 \gamma, \quad \omega^{D}(r)=\left[\omega^{R}(r)\right]^{2}
$$

In this work we take $\omega^{R}(r)$ as

$$
\omega^{R}(r)= \begin{cases}1-r / r_{c}, & \text { if } r<r_{c} \\ 0, & \text { otherwise. }\end{cases}
$$

this preserves the hydrodynamics of the system and follows from the fluctuation-dissipation theorem.

The conservative force is given by

$$
\boldsymbol{F}_{i j}^{C}= \begin{cases}A_{i j}\left(1-r_{i j} / R_{i j}\right) \hat{\boldsymbol{r}}_{i j}, & \text { if } r_{i j}<R_{i j} \\ 0, & \text { otherwise }\end{cases}
$$

where $A_{i j}$ is the interaction strength and $R_{i j}$ the cut-off distance.

The coarse-grained mapping used here for SLES is shown in Fig 1 where the parameters are taken from Anderson et al. ${ }^{38,39}$ In this model, the DPD bead represents on average 1-3 "heavy atoms" (i.e. atoms that are not Hydrogen), with the exception of water $\left(\mathrm{H}_{2} \mathrm{O}\right)$, and the density of water corresponds to $\rho r_{c}=3$. Following Groot and Rabone, ${ }^{40}$ water is treated supermolecularly, with each water bead corresponding on average to two $\mathrm{H}_{2} \mathrm{O}$ molecules giving a mapping number, $N_{m}=2$. From this information, along with the molecular volume 
of liquid water, $v_{m} \approx 30 \AA^{3}$, we can use the relation $\rho N_{m} v_{m} \equiv 1$ to determine that $r_{c} \approx 5.65 \AA$. All lengths reported in this paper are in reduced DPD units, and the previous relations can be used to convert into real units. Note that unlike other common DPD models, both $A_{i j}$ and $R_{i j}$ are varied in this representation and these values can be found in the SI. The cosurfactant CAPB requires the parametrization of several new bead types. The cut-off values, $R_{i j}$, for the beads considered in this article were assigned following the protocol set out in Anderson et al. ${ }^{38}$ using the Durchschlag and Zipper rules for individual atom contribution to the molar volume. ${ }^{41}$ We concur with Tang et al. ${ }^{42}$ who point out that there has been no systematic approach described for the parametrisation of charged beads in the DPD literature and in the current article we have not attempted to parametrise these beads to ion dependent properties. As such, we follow their pragmatic approach we set $A_{i j} R_{i j}^{3}=25$ and $R_{i j}=1$ for all interactions involving two ionic beads or one ionic bead and water, i.e. the same as between two water beads. This choice of scaling reflects the mean-field behaviour of DPD when operated in the usual parameter regime of interest. The interactions for the amide beads with alkyl and water beads were validated by calculating the $\log P$ of ethyl propenamide (The method of obtaining $\log P$ from DPD simulation was set out in Anderson et $\left.a l .{ }^{38}\right)$. The $\log P$ of ethyl propanamide had been predicted as 0.29 via EPISuite ${ }^{43}$ and our DPD model yields $\log P=0.69$.

The bead mapping is shown below in Fig 1 and the parameters are given in the SI. For computational ease, the mass of each bead is set to unity. $\boldsymbol{F}_{i j}^{B}$ includes both bonded and angular interactions for which we employ harmonic potentials,

$$
\begin{aligned}
U_{\text {bond }} & =\frac{1}{2} k_{\text {bond }}\left(r-r_{0}\right)^{2} \\
U_{\text {angle }} & =\frac{1}{2} k_{\text {angle }}\left(\theta-\theta_{0}\right)^{2}
\end{aligned}
$$

where $k_{\text {bond }}$ and $k_{\text {angle }}$ are the bond and angle constants whilst $r_{0}$ and $\theta_{0}$ are the equilibrium bond/angle lengths respectively. In all cases $k_{\text {bond }}=150 k_{B} T, k_{\text {angle }}=5 k_{B} T$ and $\theta_{0}=180^{\circ}$, whilst $r_{0}$ (in reduced DPD units) is based on the number of heavy atoms each bead represents 
$N_{\text {heavy }}$, and given by

$$
r_{0}=0.1 N_{\text {heavy }}-0.01
$$

except in the case of $\left[\mathrm{CH}_{2} \mathrm{OCH}_{2}\right]-\left[\mathrm{CH}_{2} \mathrm{OSO}_{3}^{-}\right]$. Here the non-linear arrangement of atoms means that using equation 8 would result in an over-long $r_{0}$ with $N_{\text {heavy }}=9$ leading to $r_{0}=0.89$. Therefore, for this interaction we use a modified value of $r_{0}=0.59$ is used, as used previously by Anderson et al. ${ }^{39}$
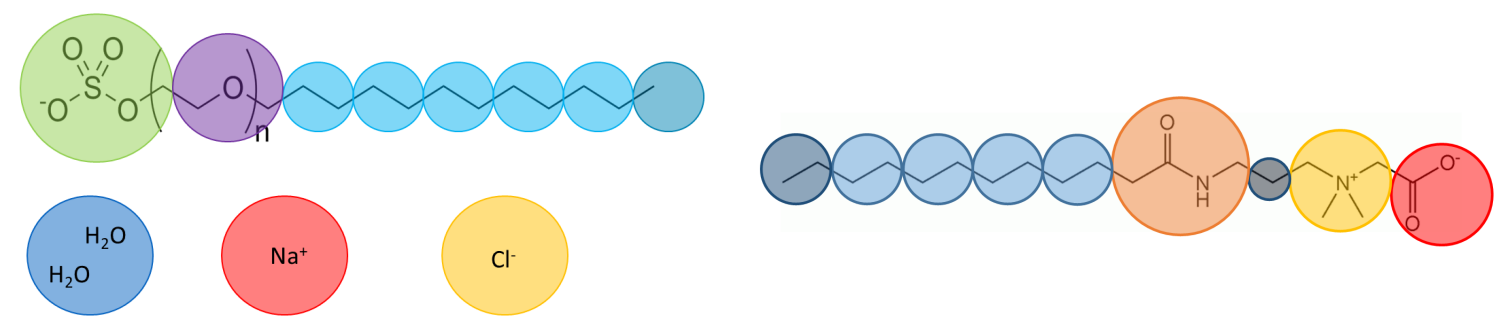

Figure 1: SLES and CAPB models and coarse-grained mapping

The final term in equation 2 is $\boldsymbol{F}_{i j}^{\text {coul }}$ and accounts for coloumbic interactions. Due to the soft nature of the DPD potential employed, $r=0$ has a finite energy. This means that point charges, as commonly used in computer simulations, would suffer from a Coulomb divergence at this point. To overcome this, we use a smeared Gaussian charge distribution as suggested by Groot ${ }^{44}$ unless otherwise specified,

$$
U_{\text {coul }}\left(r_{i j}\right)=\frac{\Gamma q_{i} q_{j}}{4 \pi r_{i j}} \operatorname{erf}\left(\frac{r_{i j}}{2 \sigma_{G}}\right)
$$

where $q_{i}, q_{j}$ are the charges on beads $i$ and $j$ respectively and $\sigma_{G}$ is the length scale of the charge (or standard deviation of the Gaussian). $\Gamma$ is the coupling constant for the reference medium, here taken to be 15.4 , and is related to the dielectric permittivity $\left(\epsilon_{r}\right)$ of the medium by 


$$
\Gamma=\frac{e^{2}}{k_{B} T \epsilon_{0} \epsilon_{r} r_{c}}
$$

where $e$ is the elementary charge and $\epsilon_{0}$ is the dielectric permittivity of vacuum. This is set using the "dielectric" command in LAMMPS or the "permittivity constant" in DL_MESO.

This model has previously been shown to accurately reproduce the phase behaviour of aqueous solutions of $\mathrm{SLES}_{1} \mathrm{EO}^{2,45}$

\section{Umbrella sampling}

In order to calculate a free energy difference, a suitable reaction coordinate, $\lambda$, needs to be defined. For example, a common reaction coordinates is the distance between two particles or micelles. ${ }^{46}$ Wang et $a l^{27}$ recently investigated several reaction coordinates for calculation the scission energy of WLM and found that one based on the number of tail beads $\left(N_{\text {tail }}\right)$ in a scission region $\left(v_{s c i}\right)$ is the most robust (Fig 2). Thus, $\lambda$ depends on the Cartesian coordinates of bead $i$, where $i$ is a tail bead as

$$
\lambda\left(\left\{x_{i}, y_{i}, z_{i}\right\}\right)=\sum_{i=1}^{N_{\text {tail }}} \omega\left(x_{i}, y_{i}, z_{i}\right)
$$

where $\omega\left(x_{i}, y_{i}, z_{i}\right)$ is a differentiable smearing function of whether bead $i$ is found in $v_{s c i}$. Here the WLM is oriented parallel to the $z$-axis and so $v_{s c i}$ is simply the volume of the simulation box slice along the z-axis (i.e. it doesn't depend on $x, y$ ) and

$$
\omega\left(x_{i}, y_{i}, z_{i}\right)=\omega\left(z_{i}\right)=\int_{z l}^{z u} K\left(z, z_{i}, \sigma\right) d z
$$

where $z l$ and $z u$ are the lower and upper limits for the scission region respectively and are given by $z_{0} \pm d$ and

$$
K\left(z, z_{i}, \sigma\right)=\frac{1}{\sqrt{2 \pi} \sigma} \exp \left(\frac{-\left(z-z_{i}\right)^{2}}{2 \sigma^{2}}\right)
$$

where $\sigma=0.01$ is a smearing parameter. The choice of $z_{0}$ is arbitrarily taken to be the 
centre of the simulation box.

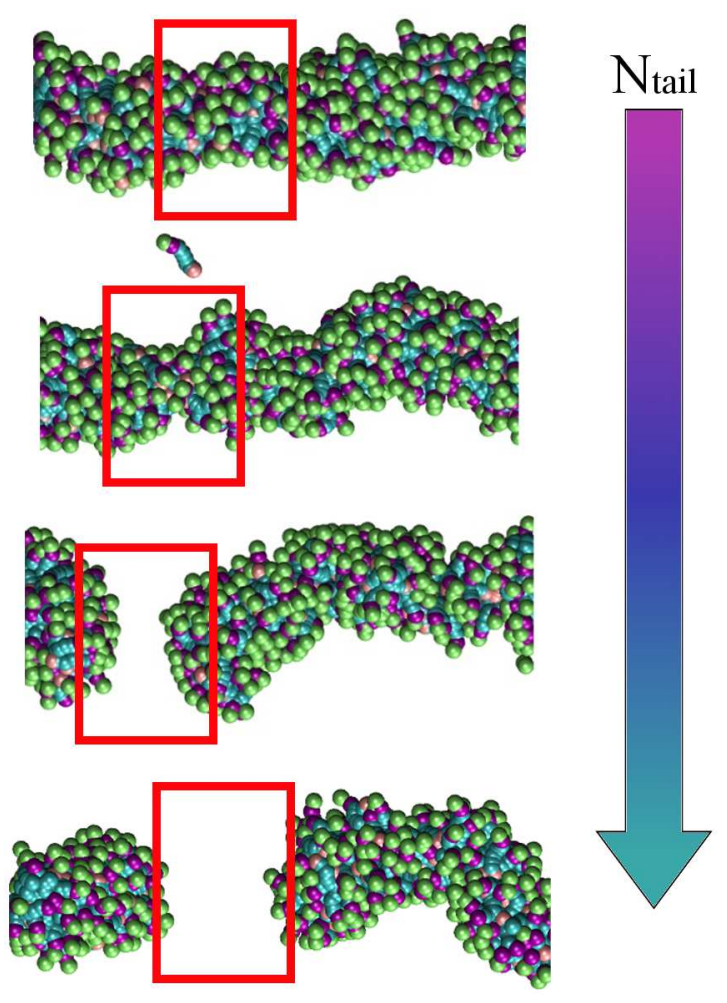

Figure 2: Representative configurations of wormlike micelle during umbrella sampling. the scission region is indicated by a red box in each case

The umbrella sampling method applies a harmonic bias on $\lambda$ with a force constant of $k_{\text {bias }}$, using to sample the free energy surface in windows of 6 tail beads, i.e. one surfactant. ${ }^{47}$ The bias is then removed using a weighted histogram analysis method (WHAM) ${ }^{48}$ and the unbiased potential of mean force (PMF) is obtained (Fig 3). Error bars for the PMF are calculated using a bootstrap procedure. ${ }^{49}$ The PMF obtained are of the form shown in Fig 3 and is consistent with that found by both Wang et al and Mandal et al ${ }^{50}$ who have shown that it is reversible with respect to fusion/fission with no activation energy. The shape of the PMF can be understood in the following way; the infinitely long WLM is the ground state (here at 402 beads or 67 surfactants). To the right of the graph with more tail beads in the scission region, the WLM is more dense which increases the free energy slightly due to both the soft repulsive interactions and the coulombic repulsion between head groups. As the number of tail beads are decreased (moving to the left of the plot), the free energy rises 
smoothly in a parabolic fashion. When the scission occurs, the free energy plateaus as the two ends of the WLM are forced apart and the solvent fills $v_{s c i}$. We did not see an activation energy, rather there is a small drift in the PMF due to the comparatively large $v_{s c i}(d=3)$ compared to the actual scission event (e.g. the formation of the two spherical caps from the intact worm-like micelle) which occurs over a much smaller area. This means that after scission has occurred there are still tail beads in the scission region and their removal due to the biasing potential, keeps on changing the value of the PMF. We show that the PMF obtained is independent of the $v_{s c i}$ for $d>2.0$, shown in the SI. At very low number of tail beads, the finite size of the simulation box causes a second, sharper drop which is consistent with the literature. ${ }^{27,50}$ Here $E_{s c i}$ is taken as the energy at scission to allow for comparison across the different systems, removing the influence of the small drift in energy.

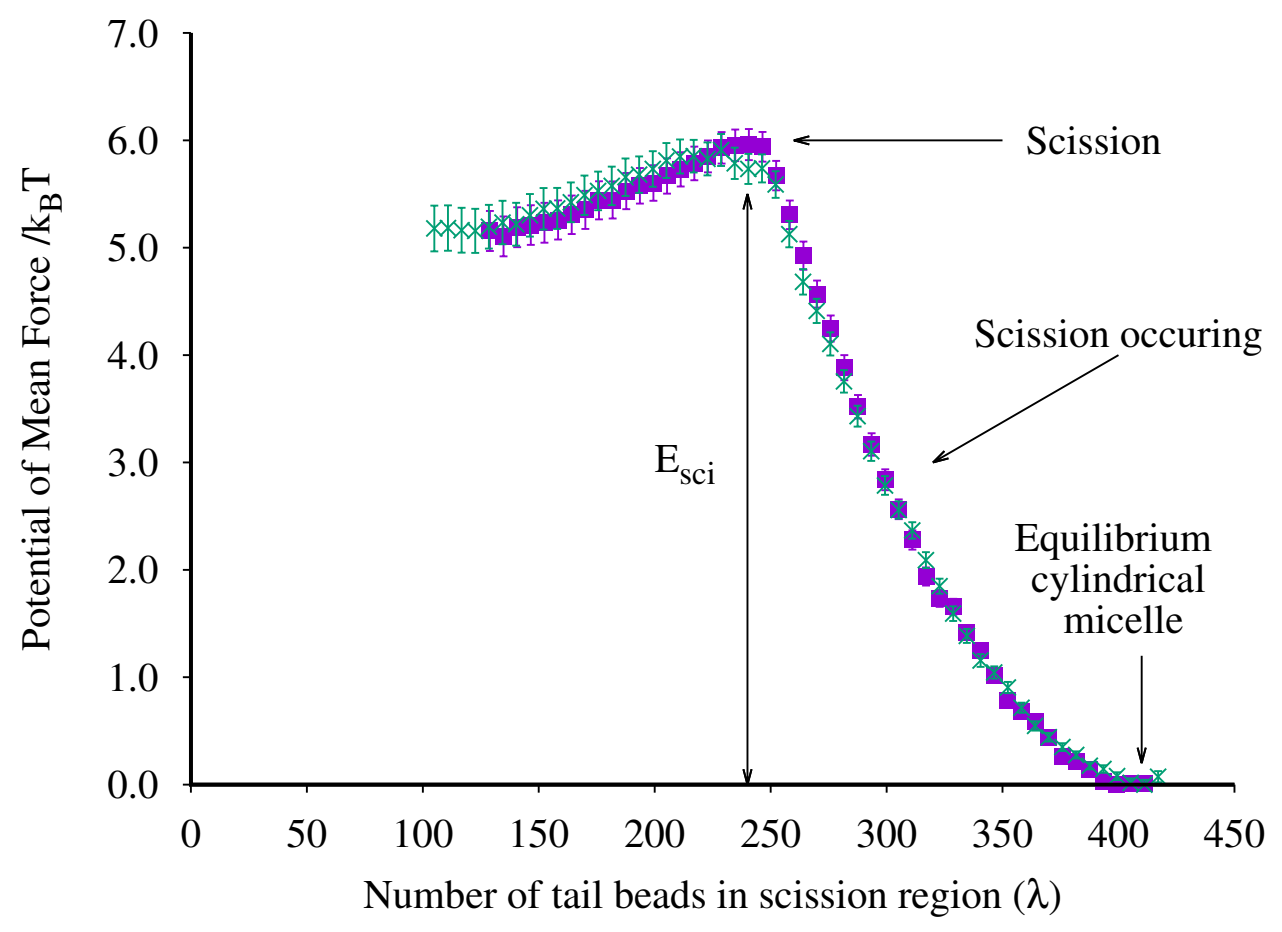

Figure 3: PMF for 5\% NaCl with 10\% (purple) and 13\% (green) SLES $_{1}$ EO. 


\section{Simulation details}

\section{Pure SLES scission energy calculations}

The simulations were run using LAMMPS ${ }^{51}$ with the PLUMED add-on ${ }^{52}$ to perform the umbrella sampling. We use a standard velocity-Verlet algorithm to integrate the simulation through time ${ }^{53}$ and periodic boundary conditions in three dimensions. The long range electrostatic forces are calculated using a standard Ewald approach of splitting the contribution into a short-range real-space and a long-range Fourier space contribution, with the longrange contribution is identical to that of point charges. Therefore, by setting the real-space contribution to zero and carefully choosing of the surrogate charge used for the inverse-space calculation (gewald flag in LAMMPS), we can successfully model the smeared charges.

Starting configurations were initially produced by packing the surfactants randomly into a cylinder aligned along the $z$-axis using PACKMOL ${ }^{54}$ and surrounded by the required number of water and ion beads to satisfy the density constraints. A short simulation is run at constant number of particles, volume and temperature $(N V T)$ with the terminal $\mathrm{CH}_{3}$ groups fixed in position to allow the surface of the micelle to equilibrate and remove any high energy head-head interactions. This is followed by a longer simulation at constant pressure $(N P T)$ in which the equilibrium number of tail beads $\left(\lambda_{=}\right)$in the scission region is calculated. The pressure is controlled using a Nose-Hoover ${ }^{55}$ barostat, coupled in the $x y$ dimensions. By allowing the $z$-direction to vary independently a tensionless WLM is maintained. To produce the starting configurations for the umbrella sampling a steered simulation was run gradually decreasing $\lambda_{0}$ from $\lambda_{0}=\lambda_{=}+3$ to $\lambda_{0}=0$ with $k_{\text {bias }}=1.0$ with uniformly spaced windows with intervals of one surfactant. In order to accurately calculate $E_{s c i}$ a lower value of $k_{\text {bias }}=0.1$ is employed for the production run for $1.5 \times 10^{6} \Delta t$ with $\Delta t=0.01$ and ensemble averages taken over the final $5 \times 10^{5} \Delta t$. To confirm that the simulation approach is not artificially stabilizing the WLM we ran a series of rigorous tests on the simulation set-up, including the box-length, length of run and size of scission region for a system of $10 \% \mathrm{SLES}_{1} \mathrm{EO}$ with $5 \%$ 
$\mathrm{NaCl}$. We compared three box sizes with $N_{\text {surf }}=375,562,750$ which correspond to a mean box length of 33, 49 and 61 DPD units respectively. All except the smallest system show the same $E_{s c i}$ (Fig 4), indicating that the micelles are not being artificially stabilised by the finite system size. Similar analysis was performed for the scission region size and length of equilibration/production runs (See SI for more information).

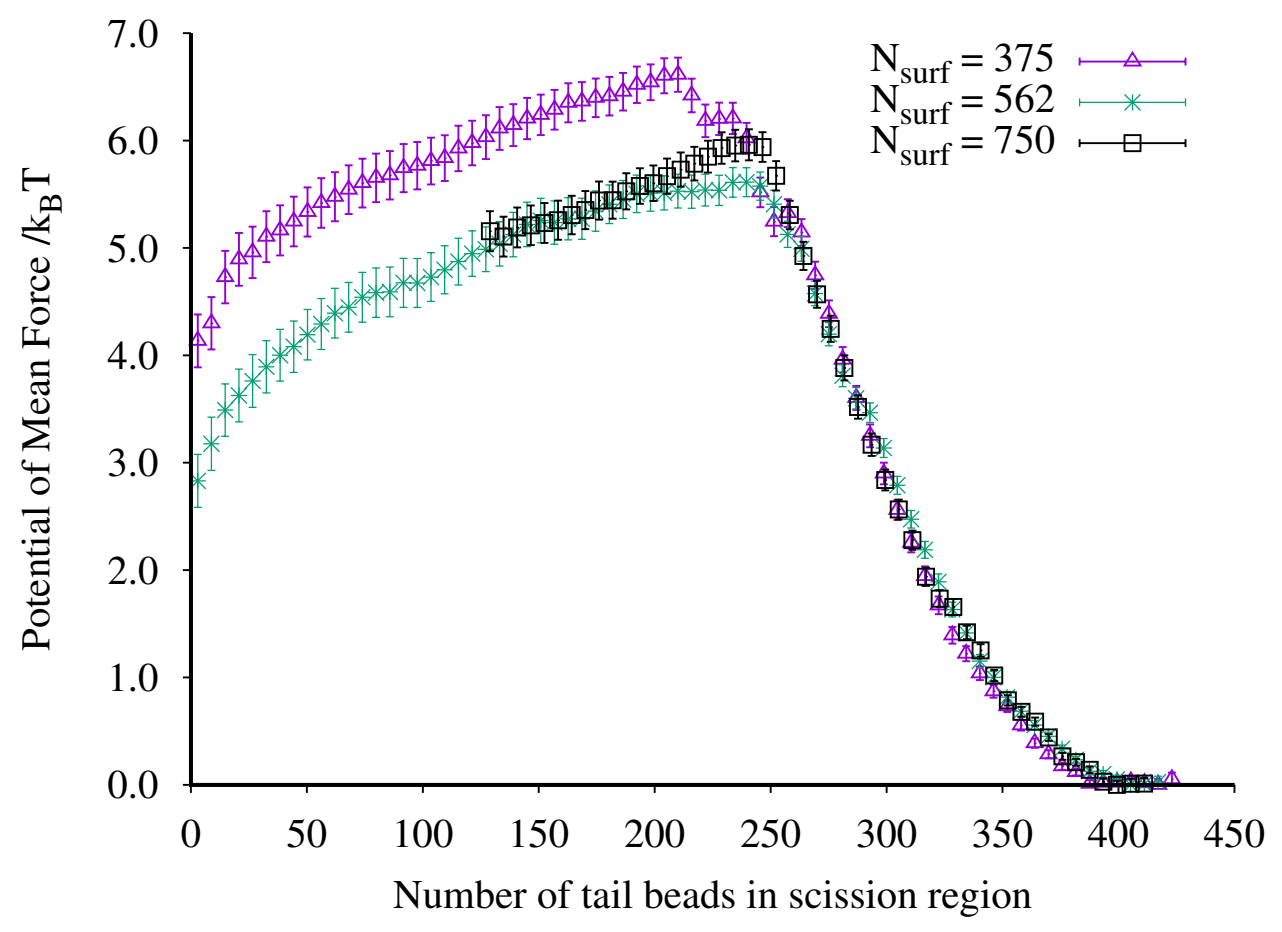

Figure 4: $\mathrm{PMFs}$ for $5 \% \mathrm{NaCl}$ with $10 \% \mathrm{SLES}_{1} \mathrm{EO}$. for $N_{\text {surf }}=375$ (purple triangle), $N_{\text {surf }}=$ 562 (green star) and $N_{\text {surf }}=750$ (black square)

Following these tests, the simulations were run with $N_{\text {surf }}=750, N_{\text {tot }}=67500$ and $d=3.0$ where $N_{\text {surf }}$ is the number of surfactant molecules. For $\mathrm{SLES}_{1} \mathrm{EO}$ this corresponds to a surfactant concentration of $10 \% \mathrm{wt}$.

Preliminary simulations were also conducted to investigate the effect of the total percentage of surfactant, with simulations run at $10 \%\left(N_{\text {surf }}=750\right)$ and $13 \%\left(N_{\text {surf }}=975\right)$ of SLES $_{1} \mathrm{EO}$ with $5 \% \mathrm{NaCl}$. It was found that both gave the same PMF (Fig 3). This can be explained by considering the simulation set-up. Essentially, we are considering an isolated micelle as the $x, y$ directions are large enough that the micelle does not interact with itself around the 
periodic boundary conditions. The law of mass action states that the more mass, the longer the micelle, both Fig 5 and Fig 4 confirm that $E_{s c i}$ is independent of micelle length and thus we expect the same value. Of course, if we consider salt:water ratio, $N_{\mathrm{NaCl}} / N_{\mathrm{H}_{2} \mathrm{O}}$, there is a negligible difference between the two systems, $N_{\mathrm{NaCl}} / N_{\mathrm{H}_{2} \mathrm{O}}=0.061$ or 0.059 for $13 \%$ and $10 \%$ respectively, given that the total number of beads is fixed. We confirm that these are the same systems by calculating the micelle contour length, $L$, and the ratio $L / N_{\text {surf }}$ which in both cases is 0.11 . Guo et $a l^{56}$ report that the local ion concentration around the micelle is important, therefore we can also consider the ratio between the total available $\mathrm{Na}^{+}$ counterions (from both added $\mathrm{NaCl}$ and SLES) and $L$, and find it to be comparable in both system sizes, (0.040 and 0.032 for $13 \%$ and $10 \%$ SLES respectively).

The simulations performed are given in Table 1.

Table 1: Simulations performed for $\mathrm{SLES}_{\mathrm{n}} \mathrm{EO}$

\begin{tabular}{cc}
\hline $\mathrm{n}$ & Percentage of $\mathrm{NaCl}$ \\
\hline 1 & $5.0,6.0,7.5,9.0$ \\
2 & $5.0,6.0,7.5,8.0,9.0,10.0,11.0,11.5,13.0,14.0,15.0$ \\
3 & $7.5,10.0,12.0,13.0,14.0,15.0$ \\
\hline
\end{tabular}

\section{Mixed surfactant phase behaviour}

All simulations investigating the phase behaviour of SLES:CAPB mixed surfactant systems were performed in NVT ensemble using DL_MESO mesoscale simulation package ${ }^{57}$ and all the systems were simulated in a cubic box with volume of $64 \times 10^{3}$ DPD unit length. Slatertype charge smearing was applied to the systems, as in previous works. ${ }^{2,34}$ For Slater-type charge smearing, the charge is represented by; ${ }^{58}$

$$
U_{\text {coul }}^{\text {Slater }}\left(r_{i j}\right)=\frac{\Gamma q_{i} q_{j}}{4 \pi r_{i j}}\left[1-\left(1+\frac{5 r_{c} r_{i j}}{8 \lambda_{s}}\right) \exp \left(-5 r_{c} r_{i j} / 4 \lambda_{s}\right)\right]
$$

where $\Gamma$ is given in equation 10 and $\lambda_{s}=1.076$ is the decay length of the charge. ${ }^{38,39} \mathrm{We}$ confirm that the two charge smearing representations, that is the Gaussian-type in equation 
9 and Slater-type in equation 14, give the same phase behaviour and does not affect the results (see SI). The simulations were run for a total of 5 million timesteps of 0.02 DPD time units and the composition of the systems are detailed in the SI. The cluster analysis was performed using UMMAP SOFTWARE. ${ }^{59}$

\section{Mixed surfactant scission energy calculations}

To investigate the effect of CAPB on the scission energy of SLES WLM we performed simulations with $10 \% \mathrm{SLES}_{1} \mathrm{EO}\left(N_{S L E S}=750\right)$ and $2 \% \mathrm{CAPB}\left(N_{C A P B}=150\right)$, with $3 \%$ and $4 \% \mathrm{NaCl}$. The same conditions were used as for the pure SLES $E_{s c i}$ calculations. Here the reaction coordinate used to calculate $E_{s c i}$ for these mixed composition micelles uses the total number of tail beads, i.e. the combined total from SLES and CAPB. This ensures that there is no additional bias on the surfactant composition of the end caps.

\section{Analysis}

\section{Contour length}

The contour length $(L)$ is calculated by splitting the simulation cell into $n_{j}$ slices along the $z$-axis of width $d r$. The centre of mass (COM) of the micelle in slice $j,\left(X_{j}, Y_{j}, Z_{j}\right)$, is then calculated with the COM in the $z$-direction taken as $L_{z} * j / n_{j}-d r / 2$. The distance between successive slabs is calculated. If the magnitude of the distance is greater than $L_{x} / 2$ (or $L_{y} / 2$ ) the micelle is around the periodic boundary conditions and the coordinates of the surfactants are unwrapped. The sum of the distances between successive slab COMs is taken as the contour length.

\section{Density plots}

The probability density, $P(r)$, for the distance from the centre of the micelle for each bead type is calculated and averaged over time, giving a one-dimensional distribution. This procedure is analogous to calculating the radial distribution function, $g_{\alpha, \beta}$, where $\alpha$ is the 
closest point to the COM contour traced by the procedure described previously and $\beta$ is the bead type of interest. Thus $P(r)$ takes into account the increasing volume with increasing distance, $r$. Note that it uses the centre of the beads in all cases. The micelle radius is taken as the mean distance of the head group (i.e. $\left[\mathrm{CH}_{2} \mathrm{OSO}_{3}{ }^{-}\right]$for SLES) from the centre of the micelle.

\section{Shannon Entropy of Mixing}

The Shannon entropy of mixing, $S_{m i x}$, can be used as a measure of disorder in the system to identify if the co-surfactants (CAPB) are truly dispersed throughout the micelle. We calculate the $S_{m i x}$ following the procedure outlined by Camesasca et al. ${ }^{60}$ Briefly, the micellar volume has been divided into $n_{x y}$ bins in the $x$ and $y$ directions and in $n_{z}$ bins in the $\mathrm{z}$ direction. Then in each bin the total number of particles is measured and the probability of finding a particular type of particle in a bin is evaluated, $S_{\text {mix }}$.

$$
S_{m i x}=\frac{-1}{N_{s}} \sum_{j=1}^{N_{s}} p_{j} \sum_{c=1}^{C} p_{(c \mid j)} \ln \left(p_{(c \mid j)}\right)
$$

where $N_{s}$ is the total number of bins in the three dimensional space, $N_{s}=n_{x y}^{2} n_{z}$ and $C$ is the total number of bead types in the systems. The conditional probability of finding a specific bead type $c$ given the binning box $j$ is given by $p_{(c \mid j)}$. We take $n_{x y}=10$ and $n_{z}=20$ and calculate the Shannon entropy over 150 configurations, taking only the head groups of the SLES and CAPB molecules into account.

\section{Results and discussion}

The calculated $E_{s c i}$ for the systems listed in Table 1 is shown in Fig 5 . In general the magnitude is somewhat lower than $E_{s c i}$ values reported in the literature by Vogtt et al ${ }^{11}$ of ca. $22 k_{B} T$, Jiang et $a l^{61}$ and Wang et $a l^{27}$ of between $12 k_{B} T$ and $40 k_{B} T$ respectively. There could be several reasons for the lower magnitude of results reported here, and it is likely to be 
a complex combination of these. Firstly, the systems of interest differ, both Wang et al and Jiang et al consider industrial SLES comprised of a mixture of chain lengths and EO groups alongside additional co-surfactants (CAPB) which are known to stabilise micelle formation ( a point that will be discussed later in this paper). Increased polydispersity is also known to stabilise WLM. ${ }^{62,63}$ We performed an additional umbrella sampling calculation of for a system of $\mathrm{SLES}_{1} \mathrm{EO}$ in which $50 \%$ of the surfactants have sixteen carbons in their alkyl tail at $5.0 \% \mathrm{NaCl}$ and found that $E_{\text {sci }}$ increased to from $5.9 k_{B} T$ to $14.9 k_{B} T$ and the PMF is given in the SI. Secondly, the lower magnitude of $E_{s c i}$ found here could be due to the coarse-graining mapping scheme employed. There is no standardised mapping procedure agreed upon in the literature. The model used in these simulations has been parametrized by solubility data, and has been shown to accurately capture the phase behaviour both here and in previous publications. ${ }^{2}$ However, this model is not parametrized with the $E_{s c i}$ in mind and it could be that the coarse-graining underestimates $E_{s c i}$. Indeed, Wang et al demonstrate the the $E_{s c i}$ is highly sensitive to the mapping scheme, and they find that varying the water interaction leads the $E_{s c i}$ to vary by around $18 k_{B} T$. Given this we compare qualitative trends in the $E_{s c i}$ with literature, rather than expecting quantitative agreement. Nevertheless we can still gain valuable information from these simulations including molecular level information on the micelle structure and we will address the trends in Fig 5 with respect to salt concentration, $c_{s}$ and number of EO groups, in detail in the following subsections.

To quantify the structure of the micelles we calculate the density distribution of the different bead types as a function of the distance from the centre of the micelle which provides information on where the different groups are located within the micelle. All the micelles show similar features and an example is shown for $\mathrm{SLES}_{1} \mathrm{EO}$ with $9 \% \mathrm{NaCl}$ in Fig 6. As expected, the hydrophobic $\left[\mathrm{CH}_{3}\right]$ and $\left[\mathrm{CH}_{2} \mathrm{CH}_{2}\right]$ groups are found within the micelle, whilst the hydrophilic head group is at the surface. The distribution for head groups, that is, $\left[\mathrm{CH}_{2} \mathrm{OSO}_{3}{ }^{-}\right]$beads, and $\left[\mathrm{CH}_{2} \mathrm{OCH}_{2}\right]$ beads can be approximated with a Gaussian distribution, from which we can extract the micelle radius (see SI). However, the 


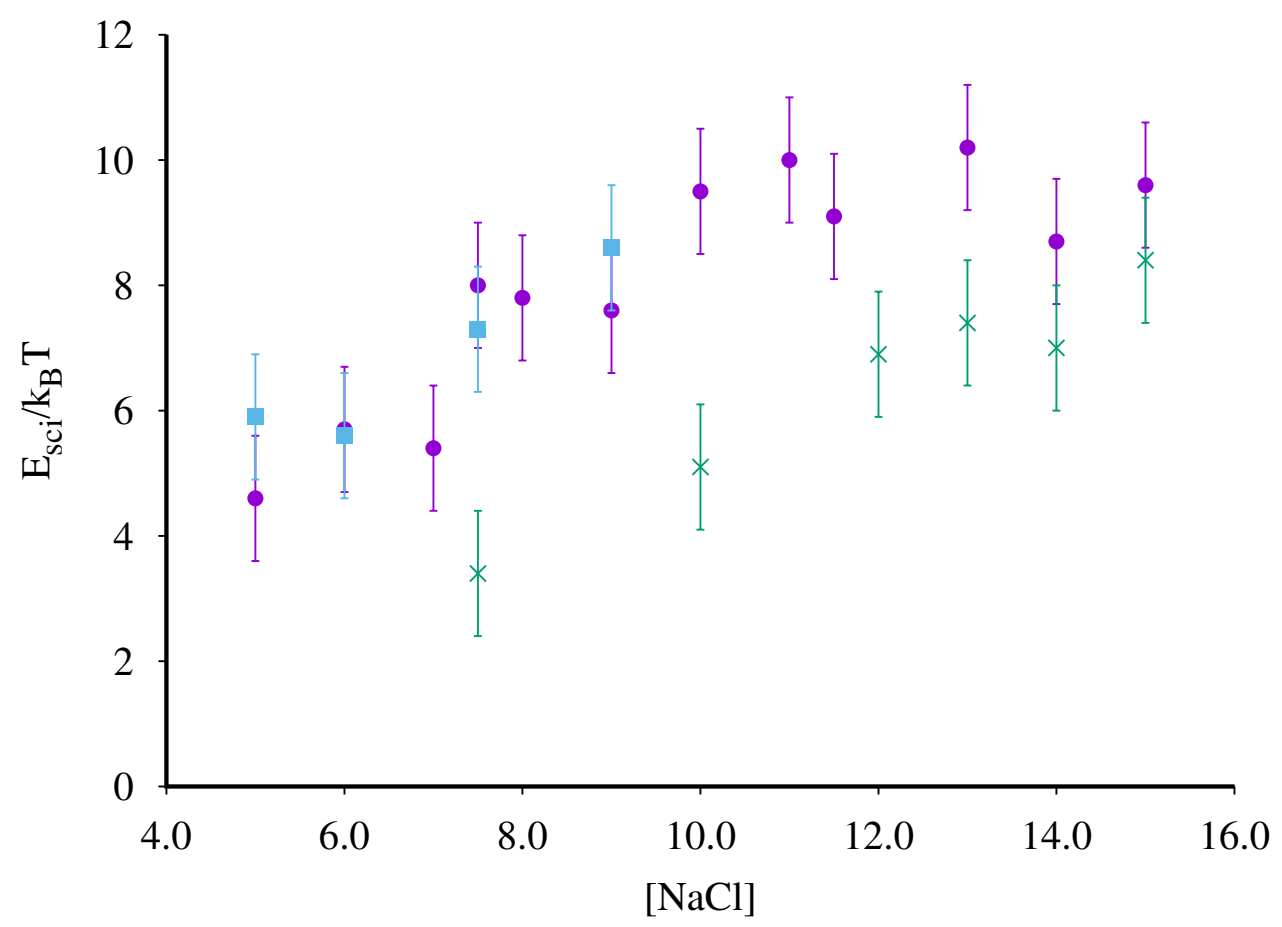

Figure 5: Calculated scission energy, $E_{s c i}$ for $\mathrm{SLES}_{\mathrm{n}} \mathrm{EO}$ as a function of salt concentration. $n=1$ corresponds to blue squares, $n=2$ to purple circles and $n=3$ to green crosses.

terminal $\left[\mathrm{CH}_{3}\right]$ bead distribution clearly shows a skewed distribution indicating that the tails are flexible rather than remaining straight and there is a non-zero probability of finding them near the head group at the surface of the micelle. The non-Gaussian distribution of $\left[\mathrm{CH}_{3}\right]$ beads highlights the importance of the tail attributes and the assumption of Gaussian distribution of the tails considered in some theoretical models. ${ }^{24,25}$

\section{Effect of salt concentration}

The $E_{s c i}$ increases with increasing salt concentration, indicating that the formation of longer micelles is favoured. In turn, this suggests that the viscosity, $\eta$, is increasing with salt concentration (see equation 1). This is in qualitative agreement with experimental results ${ }^{3}$ that show that $\eta$ increase with $\mathrm{NaCl}$ concentration up to a maximum at $7.5 \% \mathrm{NaCl}$ for $\mathrm{SLES}_{2} \mathrm{EO}$. For $\mathrm{SLES}_{2} \mathrm{EO}$ we find that $E_{s c i}$ continues to increase until $10 \% \mathrm{NaCl}$, however, two points must be considered when comparing the reported $\eta$ and the calculated $E_{s c i}$. The 


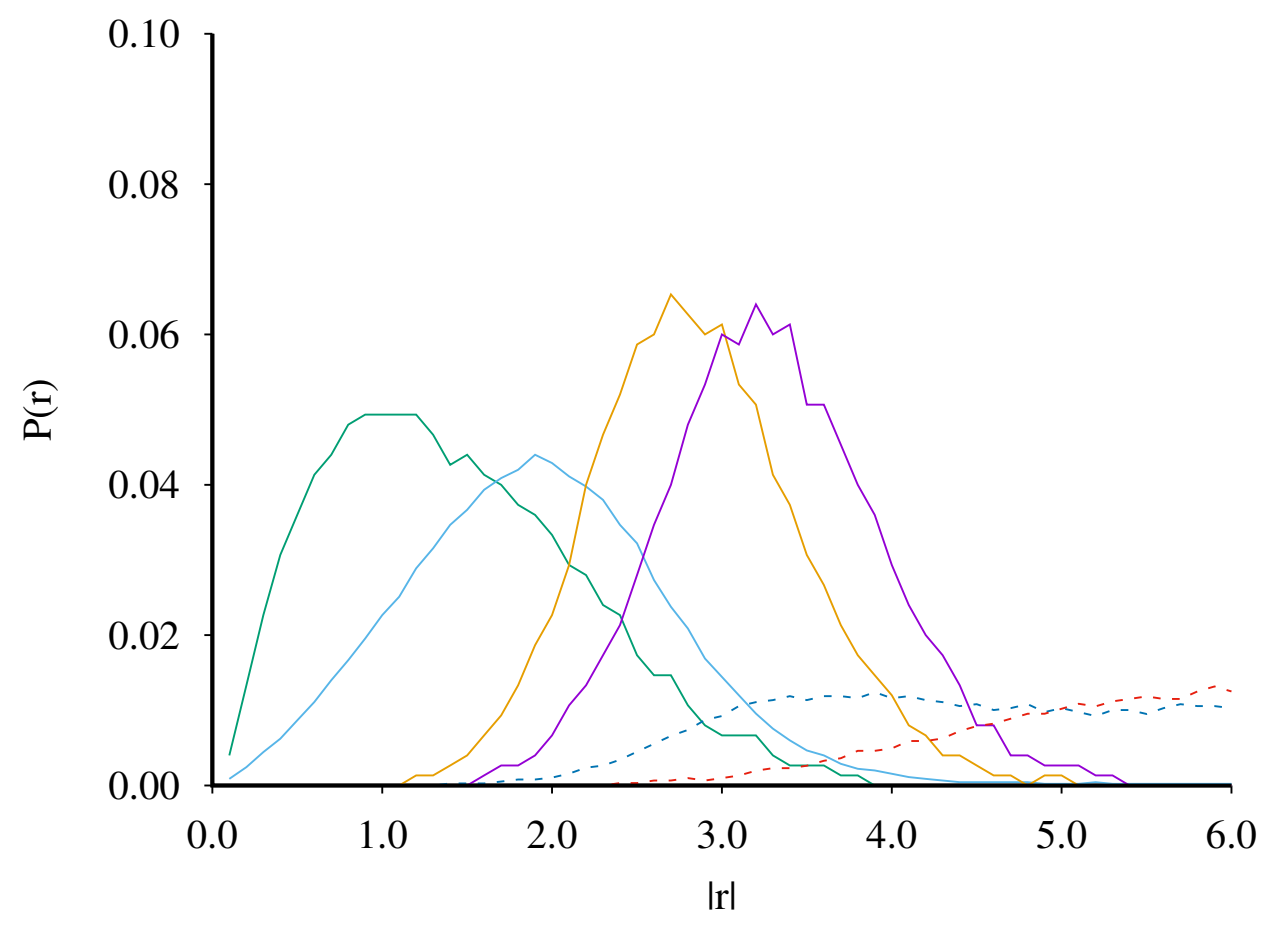

Figure 6: Probability density $(P(r))$ as a function of distance for different bead types from the centre of the micelle normal to the z-axis for $\mathrm{SLES}_{1} \mathrm{EO} 9 \% \mathrm{NaCl}$ in reduced DPD units. (Solid lines indicate beads in the surfactant molecule, green $=\left[\mathrm{CH}_{3}\right]$, blue $=\left[\mathrm{CH}_{2} \mathrm{CH}_{2}\right]$, orange $=\left[\mathrm{CH}_{2} \mathrm{OCH}_{2}\right]$ purple $=\left[\mathrm{CH}_{2} \mathrm{OSO}_{3}{ }^{-}\right]$. Dashed lines represent ions, blue $=\left[\mathrm{Na}^{+}\right]$, red $=\left[\mathrm{Cl}^{-}\right]$. )

first point is that the downturn in $\eta$ does not necessarily correspond to a decrease in $E_{s c i}$, but only shows that the free energy of producing a branched point is lower than breaking the micelle. Indeed, Mandal et $a l^{50}$ find that $E_{s c i}$ for CTAC (a cationic surfactant) continues to increase in the branched region. The second point centres around the difference between the industrial SLES samples which are produced from a natural product and are composed of a mixture of $n$ and tail lengths and pure SLES considered here. Industrially used SLES may, in addition, contain impurities such as unsulphonated alcohols which will also affect $E_{s c i}$. For $\mathrm{SLES}_{2} \mathrm{EO}$ we find a plateau in $E_{s c i}$ beginning at $10 \% \mathrm{NaCl}$ with $E_{s c i} \approx 10 k_{B} T$. To investigate if this plateau coincides with the formation of branched micelles additional simulations were run looking at the bulk behaviour. Using a cubic box with sides 40, 1920 (10\% $\left.\mathrm{SLES}_{2} \mathrm{EO}\right)$ surfactants were randomly placed into the box along with the required number of solvent beads. Note that this box size has been shown previously to be large enough to negate any 
finite size effects. ${ }^{2,34}$ An $N V T$ simulation was then run for $9 \times 10^{6} \Delta t$ for salt concentrations ranging from $8 \%$ to $15 \% \mathrm{NaCl}$. It was found that branching did not occur over the whole range. However, in a similar simulation set-up for $\mathrm{SLES}_{1} \mathrm{EO}$ we find that $E_{\text {sci }}$ increases until the concentration of $\mathrm{NaCl}$ reaches $9 \% \mathrm{NaCl}$ when branching spontaneously occurs. This result agrees with our previous work ${ }^{2}$ exploring the phase diagram of $\mathrm{SLES}_{1} \mathrm{EO}$ that finds the onset of branching at $9 \% \mathrm{NaCl}$ for a $10 \%$ surfactant concentration. The presence of branching contributes to the free energy of the system with $E_{\text {branching }}$ per branch. ${ }^{50}$ In our umbrella sampling simulations, the total number of branches fluctuates, meaning it is no longer possible to calculate $E_{s c i}$ for systems above $9 \% \mathrm{NaCl}$ as $E_{\text {branching }}$ is unknown.

To investigate the cause of the increase in $E_{s c i}$ with salt concentration, we calculate the radial distribution function, $g_{\alpha, \beta}(r)$, where $\alpha$ is the reference particle and $\beta$ the particle distribution to calculate and extract the coordination number of $\mathrm{Na}^{+}$around the $\left[\mathrm{CH}_{2} \mathrm{OSO}_{3}{ }^{-}\right]$ head group as the integral of $g(r)$ for $\mathrm{Na}^{+}$around the head groups up to the first peak at 0.85 (see SI). We find that for all values of $n$ the coordination number increases linearly with salt concentration. This can be explained by approximating the ion concentration as an "ion cloud". ${ }^{12}$ In this approximation, we consider that the ions associated with the WLM, $n_{I, a}$ are in equilibrium with the free ions in the bulk, $n_{I, f}$. Note that $n_{\mathrm{Na}^{+}, a}$ is simply the coordination number of $\mathrm{Na}^{+}$around the head groups multiplied by the number of surfactants $\left(N_{\text {surf }}\right)$, assuming that the binding is strictly 1:1. From this the change in free ions over time is given by

$$
\frac{d n_{I, f}}{d t}=-k_{a} n_{I, f} N_{s u r f}+k_{d} n_{I, a}
$$

where $k_{a}$ and $k_{d}$ are the association and dissociation constants respectively. Note that the association term depends on $N_{\text {surf }}$ because it is dependent on the number of association "sites" which scales with the size of the micelle, whilst the dissociation only depends on the number of associated ions. At equilibrium equation 16 equals zero which leads to

$$
K_{e q}=\frac{k_{d}}{k_{a}}=\frac{n_{I, f} N_{\text {surf }}}{n_{I, a}}
$$


We then follow a similar working to that in reference ${ }^{12}$ and remembering that $n_{I, 0}=n_{I, a}+n_{I, f}$ we gain

$$
n_{I, a}=\frac{N_{\text {surf }} n_{I, 0}}{K_{e q}+N_{\text {surf }}}
$$

without loss of generality for ion type. We are interested in $\mathrm{Na}^{+}$, for which we know that the total number of $\mathrm{Na}^{+}$is given by

$$
n_{\mathrm{Na}^{+}, 0}=\frac{1}{2} c_{s} N_{t o t}+N_{\text {surf }}
$$

where $c_{s}$ is the salt number fraction and $N_{\text {tot }}$ is the total number of beads. The first term in equation 19 accounts for the $\mathrm{Na}^{+}$from the additional salt, recalling that the mass of each bead is equivalent, and the second term accounts for the counter-ions from the SLES. Substituting equation 19 into 18 gives an expression relating the number of associated $\mathrm{Na}^{+}$ to the salt concentration;

$$
n_{\mathrm{Na}^{+}, a}=\frac{\frac{1}{2} c_{s} N_{\text {tot }}+N_{\text {surf }}}{\frac{K_{e q}}{N_{\text {surf } f}}+1}
$$

which shows that we expect the number of associated ions to vary linearly with salt concentration, which is shown in Fig 7 a, noting that $N_{\text {surf }}$ and $N_{\text {tot }}$ are constant.
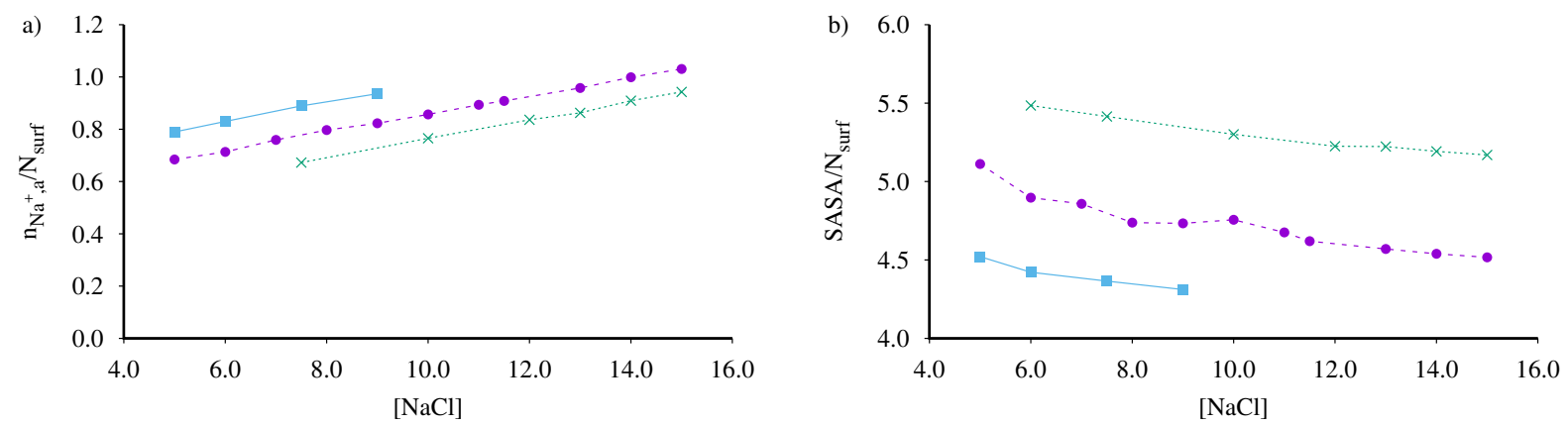

Figure 7: a) $n_{N a^{+}, a} / N_{\text {surf }}$ for SLES $\mathrm{n}$ EO $n=1$ (blue squares, solid line), $n=2$ (purple circles, dashed line) and $n=3$ (green crosses, dotted line) b) Solvent accessible surface area with a probe of size 1.0 for SLES $_{\mathrm{n}}$ EO. In all cases $n=1$ corresponds to blue squares with a solid line, $n=2$ to purple circles with a dashed line and $n=3$ to green crosses with a dashed line 
The increase in associated $\mathrm{Na}^{+}$ions screens the charged $\left[\mathrm{CH}_{2} \mathrm{OSO}_{3}{ }^{-}\right]$allowing the surfactants in the cylindrical section to pack closer together increasing the shielding of the hydrophobic core. This increased packing is evidenced by a decreasing solvent accessible surface area (SASA) shown in Fig 7b. We measure the SASA or Connolly surface using a probe of size 1.0 (i.e. the size of a water bead) and again we find that the SASA decreases with increasing salt concentration, suggesting that the head groups pack closer together.

The packing parameter model proposed by Israelachvili ${ }^{20}$ can be used to explain the relationship between surface area and $E_{s c i}$. This uses a geometrical relationship to predict the shape of aggregates formed by surfactants. The packing parameter is given by

$$
p p=\frac{v}{a_{0} l}
$$

where $v$ and $l$ are the volume and length of the hydrophobic tail respectively and $a_{0}$ is the effective head group area. The theory states that for spherical micelles, $p p=\frac{1}{3}$, whilst for (infinite) cylinders $p p=\frac{1}{2}$. Thus a value of $p p$ closer to $\frac{1}{2}$ indicates the formation of longer micelles is favoured over shorter, spherical micelles. From the surface area calculations (Fig. $7 \mathrm{~b}$ ), we can see that $a_{0}$ decreases with increasing salt concentration, therefore $p p$ increases, favouring the formation longer micelles which is consistent with a higher $E_{s c i}$ (equation 1).

We also measure the SASA for the broken WLM and find a similar trend in its value (i.e. decreasing SASA with increasing salt concentration) which can be found in the SI in agreement with a reduced $a_{0}$ and increasing $p p$ with increasing salt concentration. The SASA for the broken WLM are higher than that for the infinitely long WLM in all cases. This is consistent with a higher $p p$ for the broken worms, as predicted theoretically due to the presence of hemispherical caps. The difference in SASA between the broken and whole WLM are approximately constant across the salt concentration and equal to $1.25,1.27$ and 1.67 for $n=1,2,3$ respectively. The similarity for $n=1,2$ is consistent with the similar $E_{s c i}$ found in Fig 5, whilst $\mathrm{SLES}_{3} \mathrm{EO}$ shows a lower $E_{s c i}$. 


\section{Effect of EO groups}

We also investigate the effect that increasing the number of EO groups has on the $E_{s c i}$.

When increasing from $n=1$ to $n=2$ we see little increase in $E_{s c i}$. However, the presence of a third EO group decreases the scission energy. Indeed, preliminary simulations show that the wormlike micelles are only stable above $7.5 \% \mathrm{NaCl}$ for $n=3$, whilst for $n=1$ the range of stability is $5.0-9.0 \% \mathrm{NaCl}$. The drop in $E_{\text {sci }}$ can be explained by the increased flexibility of the head group. Using the same geometric relation reported in equation 21, a larger, more flexible head group means that the effective head group area increases and the packing parameter decreases, favouring shorter micelles with a lower $E_{s c i}$. This can be seen in the SI which shows that both the micelle radius (as expected due to the longer surfactant) and the contour length per surfactant increases with increasing $n$. However, the increase in micelle radius is significantly less than the diameter of the additional $\left[\mathrm{CH}_{2} \mathrm{OCH}_{2}\right]$ bead (1.116) with an average increase of 0.33 for $n=1$ to $n=2$ and 0.27 for $n=2$ to $n=3$. This discrepancy is due to the flexibility of the head group and demonstrates the importance of the packing parameter in relation to the effective head group area, that is, taking account of the flexibility of the head group and any bound ions associated with it. The increased flexibility of the head group also has an effect on the coordination number of $\left[\mathrm{Na}^{+}\right]$around $\left[\mathrm{CH}_{2} \mathrm{OSO}_{3}{ }^{-}\right]$, with the ion less tightly bound for $n=3$ than $n=1,2$ as can be seen in Fig $7 a$.

\section{Effect of co-surfactant}

In industrial applications a co-surfactant is often used to modify the properties of the base surfactant (in this case SLES). ${ }^{64} \mathrm{~A}$ common co-surfactant that is added to SLES is cocamidopropyl betaine, CAPB, which serves a number of purposes including as a thickener, antistatic agent and to reduce skin irritation. CAPB is a zwitterionic surfactant with a dodecyl tail, matching the length of the alkyl tail of the bulk surfactant. The addition of another surfactant changes the behaviour of the bulk system. In particular, the incorporation 
of a small amount of CAPB into pure SLES shifts the experimental salt curve to the left, i.e. WLM form at lower salt concentrations. ${ }^{65}$ The experimental salt curve plots the viscosity against salt concentration and for many anionic surfactant mixtures shows a characteristic bell-shaped curve. ${ }^{3,65}$ The increase in viscosity for these curves has been attributed in the literature to the one dimensional growth of micelles and the formation of WLM.

To ensure the validity of our CAPB model, we investigate the phase behaviour with salt concentration for a system comprising of $9.85 \%$ SLES $_{1} \mathrm{EO}$ and $1.15 \% \mathrm{CAPB}$, giving a total surfactant concentration of $11 \%$ as in the work of Tang et al. ${ }^{42}$ The effect of salt concentration on the phase behaviour for SLES:CAPB is shown in Fig 8.

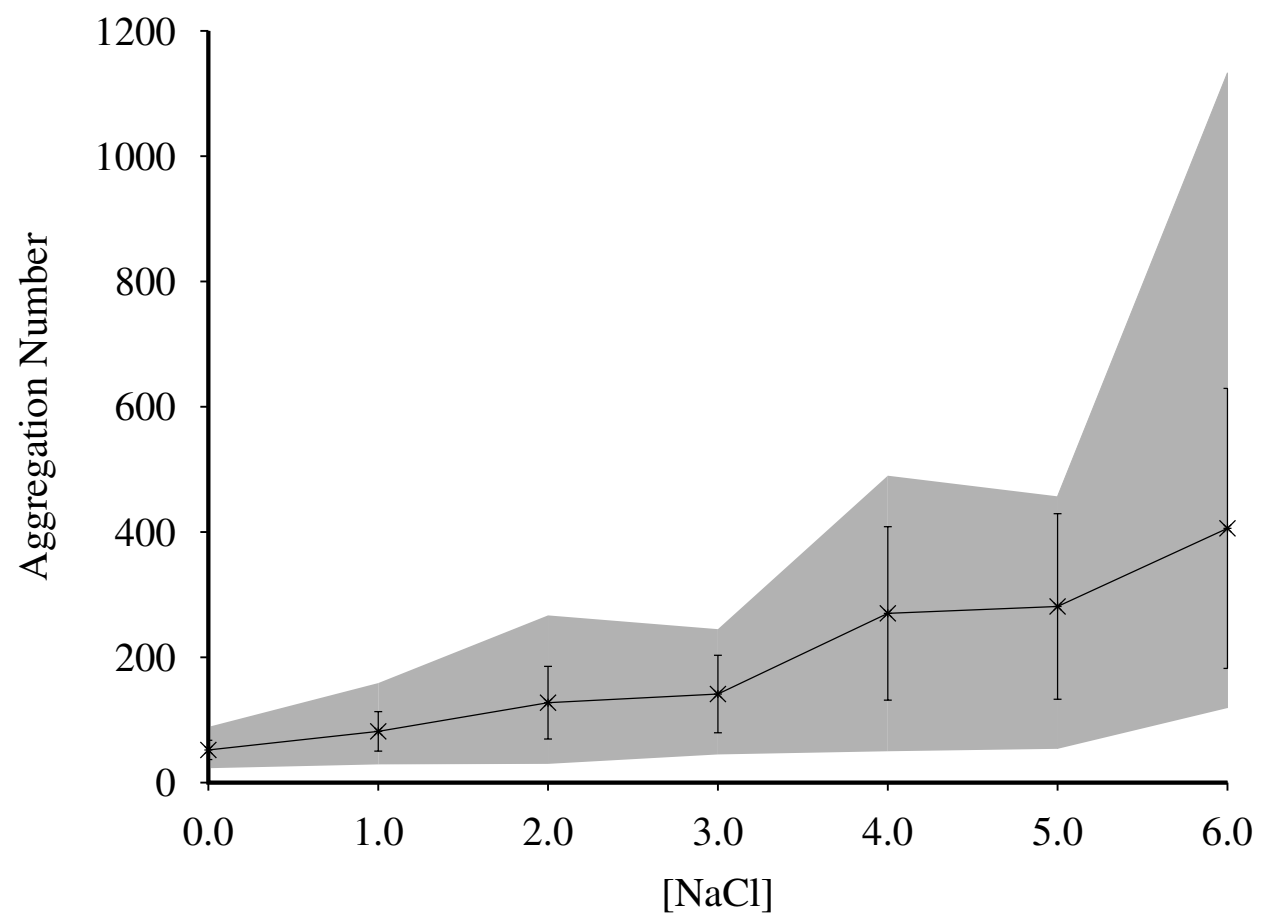

Figure 8: The mean aggregation number as a function of salt concentration for for $9.65 \%$ SLES(1)EO and 1.15\% CAPB. The error bars show the standard deviation whilst the filled area indicates the minimum and maximum micelle size. Representative snapshots can be found in the SI.

We find that at $[\mathrm{NaCl}]$ equal to $0 \%$, only spherical micelles are present. Slightly larger aggregates are formed between 1-2\%, where small rods start to emerge. At $3 \% \mathrm{NaCl}$ a transition between rods and short worms is observed; stable WLMs can be found at 4, 5 
and $6 \%$ salt concentration. These results are consistent with the experimental data reported by Tang et al. ${ }^{42}$ where increase in viscosity is observed at a salt concentration larger than $1.5 \%$.

We then go on to calculate the $E_{s c i}$ for a system comprising of $10 \%$ SLES $_{1}$ EO with $2 \%$ CAPB. WLM were found to form at a much lower salt concentration, $3 \% \mathrm{NaCl}$, than for pure $\mathrm{SLES}_{1} \mathrm{EO}$ systems that only showed stable WLM above $5 \% \mathrm{NaCl}$, in agreement with the bulk phase behaviour observed. In this case with CAPB co-surfactant spontaneous branching was seen at $5 \% \mathrm{NaCl}$ and above. The zwitterionic CAPB head groups effectively increase the distance between neighbouring negatively charged SLES head groups, thus reducing the repulsion between them and promoting a closer packing. The $E_{s c i}$ for WLM observed at $3 \%$ and $4 \% \mathrm{NaCl}$ is $4.8 k_{B} T$ and $5.5 k_{B} T$ respectively.

To confirm that the CAPB is randomly distributed throughout the micelles we calculate the Shannon entropy, $S_{m i x}$, for the systems simulated and compare it with two limiting cases: the first in which the CAPB were randomly distributed throughout the micelle and the second with complete phase separation between the SLES and CAPB. The total Shannon entropy of the demixed state was $0.0199 \pm 0.0095$, whilst for the completely mixed state it was $0.7121 \pm .0874$. The calculated $S_{m i x}$ was $0.5910 \pm 0.0957$ and $0.6003 \pm 0.1002$ for $3.0 \%$ and $4.0 \% \mathrm{NaCl}$ respectively for the whole WLM and $0.5678 \pm 0.1019$ and $0.5048 \pm 0.0885$ for the broken WLM at $3 \%$ and $4 \% \mathrm{NaCl}$ respectively. These results are close to the value for the randomly mixed limiting case, and so we can deduce that there is no microphase segregation occurring. We confirm that there is no microphase segregation at the micelle end caps by calculating the ratio of SLES:CAPB, where we define the end caps as the slice along $z$ with no surfactants present $\pm 10 r_{c}$. We find that the ratio SLES:CAPB is $0.202 \pm 0.017$ and $0.218 \pm 0.042$ for $3 \%$ and $4 \% \mathrm{NaCl}$ respectively, both of which are close to the global ratio (0.2), thus confirming that there is no local enrichment of CAPB at the end caps. 


\section{Conclusions}

In conclusion, we have calculated the scission energy, $E_{\text {sci }}$, of wormlike micelles comprised of monodisperse $\mathrm{SLES}_{\mathrm{n}} \mathrm{EO}$ both with and without the presence of a co-surfactant, CAPB and investigated the effect of salt concentration and number of EO groups. We find that $E_{s c i}$ and thus the mean micelle length increases with salt concentration in agreement with a higher experimental viscosity. This increase in $E_{s c i}$ is dominated by the electrostatic interactions. As the salt level increases it screens the repulsion between the negatively charged head groups which can therefore pack closer together. We rationalise this using an ion-cloud analogy and the packing parameter approach of Israelachvili. ${ }^{20}$ The opposite trend is seen with increasing number of EO groups $(n)$. Whilst $n=1$ and $n=2$ give similar $E_{s c i}, n=2$ has a larger range of stability for wormlike micelles than $n=1$. The addition of a 3rd EO group decreases $E_{s c i}$ which is again explained using a packing parameter approach. The addition of CAPB leads to stable WLM at a lower salt concentration than for pure SLES micelles, as the zwitterionic head groups reduce the repulsion between $\left[\mathrm{CH}_{2} \mathrm{OSO}_{3}{ }^{-}\right]$beads. Whilst the resulting scission energies are lower than those reported in the experimental literature, we ascribe this discrepancy to three possible causes which we investigate. The first is the monodisperse nature of our systems. We suggest that tail length polydispersity stabilises WLM as they interrupt the regular packing of monodisperse tails. The second is the possibility that the simulation set-up is artificially stabilizing unstable micelles. We address this through a series of simulations investigating the different aspects of the set-up and find consistent results for the $E_{s c i}$. Finally, the low order of magnitude may be due to the coarse-grained and soft nature of the model which was not developed with the $E_{s c i}$ as a target function and has been shown to accurately reproduce the phase behaviour. Nevertheless the qualitative trends and molecular level information provided here are important to understanding the fundamental factors that influence the $E_{s c i}$ and thus the average micelle length and physical properties of these micellar solutions. Further work into the mechanism of scission is required to fully understand the dynamics of the system, 
however that is beyond the scope for this work.

\section{Acknowledgement}

This work was carried out as part of the EPSRC and Unilever funded "CAFE4DM" Prosperity Partnership grant as part of a research consortium involving the University of Manchester, the University of Cambridge, STFC and Unilever (EP/R00482X/1). R.L. Anderson and A. Del Regno were supported by the STFC Hartree Centre's Innovation: Return on Research programme, funded by the Department for Business, Energy \& Industrial Strategy. This work used the Cirrus UK National Tier-2 HPC Service at EPCC (http://www.cirrus.ac.uk) funded by the University of Edinburgh and EPSRC (EP/P020267/1). C. R. Wand, M. Panoukidou and P. Carbone would like to acknowledge the assistance given by Research IT and the use of the Computational Shared Facility at The University of Manchester.

\section{Supporting Information Available}

Model parameters.

Preliminary simulations; the scission region size, simulation length dependence and umbrella sampling window spacing.

SLES-only systems; effect of tail polydispersity on the scission energy, micelle attributes including contour length, radius of micelle, radial distribution function of $\mathrm{Na}^{+}$and SASA of broken WLMs.

SLES:CAPB systems; simulation details, comparison of charge smearing, representatitve snapshots of phase behaviour simulations. 


\section{References}

(1) Kronberg, B.; Lindman, B. Surfactants and polymers in aqueous solution; John Wiley \& Sons Ltd., Chichester, 2003.

(2) Panoukidou, M.; Wand, C. R.; Del Regno, A.; Anderson, R. L.; Carbone, P. Constructing the phase diagram of sodium laurylethoxysulfate using dissipative particle dynamics. Journal of colloid and interface science 2019, 557, 34-44.

(3) Parker, A.; Fieber, W. Viscoelasticity of anionic wormlike micelles: effects of ionic strength and small hydrophobic molecules. Soft Matter 2013, 9, 1203-1213.

(4) Dhakal, S.; Sureshkumar, R. Anomalous diffusion and stress relaxation in surfactant micelles. Physical Review E 2017, 96, 012605.

(5) Dreiss, C. A. Wormlike micelles: where do we stand? Recent developments, linear rheology and scattering techniques. Soft Matter 2007, 3, 956-970.

(6) Shikata, T.; Hirata, H.; Kotaka, T. Micelle formation of detergent molecules in aqueous media. 2. Role of free salicylate ions on viscoelastic properties of aqueous cetyltrimethylammonium bromide-sodium salicylate solutions. Langmuir 1988, 4, 354359.

(7) Maitland, G. Oil and gas production. Current opinion in colloid \& interface science 2000, 5, 301-311.

(8) Cornwell, P. A review of shampoo surfactant technology: consumer benefits, raw materials and recent developments. International journal of cosmetic science $\mathbf{2 0 1 8}$, $40,16-30$.

(9) Thiam, A. R.; Farese Jr, R. V.; Walther, T. C. The biophysics and cell biology of lipid droplets. Nature reviews Molecular cell biology 2013, 14, 775. 
(10) Candau, S.; Khatory, A.; Lequeux, F.; Kern, F. Rheological behaviour of wormlike micelles: effect of salt content. Le Journal de Physique IV 1993, 3, C1-197.

(11) Vogtt, K.; Jiang, H.; Beaucage, G.; Weaver, M. Free Energy of Scission for Sodium Laureth-1-Sulfate Wormlike Micelles. Langmuir 2017, 33, 1872-1880.

(12) Vogtt, K.; Beaucage, G.; Weaver, M.; Jiang, H. Thermodynamic stability of worm-like micelle solutions. Soft matter 2017, 13, 6068-6078.

(13) Zou, W.; Larson, R. G. A mesoscopic simulation method for predicting the rheology of semi-dilute wormlike micellar solutions. Journal of Rheology 2014, 58, 681-721.

(14) Cates, M.; Candau, S. Statics and dynamics of worm-like surfactant micelles. Journal of Physics: Condensed Matter 1990, 2, 6869.

(15) Srinivasan, V.; Blankschtein, D. Effect of counterion binding on micellar solution behavior: 2. Prediction of micellar solution properties of ionic surfactant- electrolyte systems. Langmuir 2003, 19, 9946-9961.

(16) Grmela, M.; Chinesta, F.; Ammar, A. Mesoscopic tube model of fluids composed of worm-like micelles. Rheologica acta 2010, 49, 495-506.

(17) Cates, M. E.; Fielding, S. M. Rheology of giant micelles. Advances in Physics 2006, $55,799-879$.

(18) Missel, P. J.; Mazer, N.; Benedek, G.; Young, C.; Carey, M. C. Thermodynamic analysis of the growth of sodium dodecyl sulfate micelles. The Journal of Physical Chemistry 1980, 84, 1044-1057.

(19) Missel, P. J.; Mazer, N. A.; Benedek, G. B.; Carey, M. C. Influence of chain length on the sphere-to-rod transition in alkyl sulfate micelles. The Journal of Physical Chemistry 1983, 87, 1264-1277. 
(20) Israelachvili, J. N.; Mitchell, D. J.; Ninham, B. W. Theory of self-assembly of hydrocarbon amphiphiles into micelles and bilayers. Journal of the Chemical Society, Faraday Transactions 2: Molecular and Chemical Physics 1976, 72, 1525-1568.

(21) Israelachvili, J. N. Intermolecular and surface forces; Academic press, 2015.

(22) Tanford, C. Micelle shape and size. The Journal of Physical Chemistry 1972, 76, 30203024 .

(23) Nagarajan, R.; Ruckenstein, E. Theory of surfactant self-assembly: a predictive molecular thermodynamic approach. Langmuir 1991, 7, 2934-2969.

(24) Nagarajan, R. Molecular packing parameter and surfactant self-assembly: the neglected role of the surfactant tail. Langmuir 2002, 18, 31-38.

(25) Danov, K. D.; Kralchevsky, P. A.; Stoyanov, S. D.; Cook, J. L.; Stott, I. P.; Pelan, E. G. Growth of wormlike micelles in nonionic surfactant solutions: Quantitative theory vs. experiment. Advances in colloid and interface science 2018, 256, 1-22.

(26) Danov, K. D.; Kralchevsky, P. A.; Stoyanov, S. D.; Cook, J. L.; Stott, I. P. Analytical modeling of micelle growth. 1. Chain-conformation free energy of binary mixed spherical, wormlike and lamellar micelles. Journal of colloid and interface science 2019, 547, 245-255.

(27) Wang, H.; Tang, X.; Eike, D. M.; Larson, R. G.; Koenig, P. H. Scission Free Energies for Wormlike Surfactant Micelles: Development of a Simulation Protocol, Application, and Validation for Personal Care Formulations. Langmuir 2018, 34, 1564-1573.

(28) Petekidis, G.; Galloway, L.; Egelhaaf, S.; Cates, M.; Poon, W. Mixtures of colloids and wormlike micelles: Phase behavior and kinetics. Langmuir 2002, 18, 4248-4257.

(29) Aoudia, M.; Al-Maamari, T.; Al-Salmi, F. Intramolecular and intermolecular iondipole interactions in sodium lauryl ether sulfates (SLES) self-aggregation and mixed 
micellization with Triton X-100. Colloids and Surfaces A: Physicochemical and Engineering Aspects 2009, 335, 55-61.

(30) Aoudia, M.; Al-Haddabi, B.; Al-Harthi, Z.; Al-Rubkhi, A. Sodium lauryl ether sulfate micellization and water solubility enhancement towards naphthalene and pyrene: effect of the degree of ethoxylation. Journal of surfactants and detergents 2010, 13, 103-111.

(31) Hoogerbrugge, P.; Koelman, J. Simulating microscopic hydrodynamic phenomena with dissipative particle dynamics. EPL (Europhysics Letters) 1992, 19, 155.

(32) Prhashanna, A.; Khan, S. A.; Chen, S. B. Micelle morphology and chain conformation of triblock copolymers under shear: LA-DPD study. Colloids and Surfaces A: Physicochemical and Engineering Aspects 2016, 506, 457-466.

(33) Droghetti, H.; Pagonabarraga, I.; Carbone, P.; Asinari, P.; Marchisio, D. Dissipative particle dynamics simulations of tri-block co-polymer and water: Phase diagram validation and microstructure identification. The Journal of chemical physics 2018, $149,184903$.

(34) Johnston, M. A.; Swope, W. C.; Jordan, K. E.; Warren, P. B.; Noro, M. G.; Bray, D. J.; Anderson, R. L. Toward a standard protocol for micelle simulation. The Journal of Physical Chemistry B 2016, 120, 6337-6351.

(35) Lee, M.-T.; Vishnyakov, A.; Neimark, A. V. Calculations of critical micelle concentration by dissipative particle dynamics simulations: the role of chain rigidity. The Journal of Physical Chemistry B 2013, 117, 10304-10310.

(36) Vishnyakov, A.; Lee, M.-T.; Neimark, A. V. Prediction of the critical micelle concentration of nonionic surfactants by dissipative particle dynamics simulations. The journal of physical chemistry letters 2013, 4, 797-802. 
(37) Espanol, P.; Warren, P. Statistical mechanics of dissipative particle dynamics. EPL (Europhysics Letters) 1995, 30, 191.

(38) Anderson, R. L.; Bray, D. J.; Ferrante, A. S.; Noro, M. G.; Stott, I. P.; Warren, P. B. Dissipative particle dynamics: Systematic parametrization using wateroctanol partition coefficients. The Journal of chemical physics 2017, 147, 094503.

(39) Anderson, R. L.; Bray, D. J.; Del Regno, A.; Seaton, M. A.; Ferrante, A. S.; Warren, P. B. Micelle Formation in Alkyl Sulfate Surfactants Using Dissipative Particle Dynamics. Journal of chemical theory and computation 2018, 14, 2633-2643.

(40) Groot, R. D.; Rabone, K. Mesoscopic simulation of cell membrane damage, morphology change and rupture by nonionic surfactants. Biophysical journal 2001, 81, 725-736.

(41) Durchschlag, H.; Zipper, P. Calculation of the partial volume of organic compounds and polymers. Progress in Colloid and Polymer Science 1994, 94, 20-39.

(42) Tang, X.; Zou, W.; Koenig, P. H.; McConaughy, S. D.; Weaver, M. R.; Eike, D. M.; Schmidt, M. J.; Larson, R. G. Multiscale modeling of the effects of salt and perfume raw materials on the rheological properties of commercial threadlike micellar solutions. The Journal of Physical Chemistry B 2017, 121, 2468-2485.

(43) Pence, H. E.; Williams, A. ChemSpider: an online chemical information resource. 2010.

(44) Groot, R. D. Electrostatic interactions in dissipative particle dynamics?simulation of polyelectrolytes and anionic surfactants. The Journal of chemical physics 2003, 118, $11265-11277$.

(45) Eslami, H.; Khani, M.; Muller-Plathe, F. Gaussian charge distributions for incorporation of electrostatic interactions in dissipative particle dynamics: Application to self-assembly of surfactants. Journal of chemical theory and computation 2019, 15, 4197-4207. 
(46) Sangwai, A. V.; Sureshkumar, R. Binary interactions and salt-induced coalescence of spherical micelles of cationic surfactants from molecular dynamics simulations. Langmuir 2011, 28, 1127-1135.

(47) Frenkel, D.; Smit, B. Understanding molecular simulation: from algorithms to applications; Elsevier, 2001; Vol. 1.

(48) Grossfield, A. WHAM: the weighted histogram analysis method. version 2012, 2, 06.

(49) Efron, B.; Tibshirani, R. J. An introduction to the bootstrap; CRC press, 1994.

(50) Mandal, T.; Koenig, P. H.; Larson, R. G. Nonmonotonic Scission and Branching Free Energies as Functions of Hydrotrope Concentration for Charged Micelles. Physical review letters 2018, 121, 038001.

(51) Plimpton, S. Fast parallel algorithms for short-range molecular dynamics. Journal of computational physics 1995, 117, 1-19.

(52) Tribello, G. A.; Bonomi, M.; Branduardi, D.; Camilloni, C.; Bussi, G. PLumeD 2: New feathers for an old bird. Computer Physics Communications 2014, 185, 604-613.

(53) Verlet, L. Computer" experiments" on classical fluids. I. Thermodynamical properties of Lennard-Jones molecules. Physical review 1967, 159, 98.

(54) Martínez, L.; Andrade, R.; Birgin, E. G.; Martínez, J. M. PACKMOL: a package for building initial configurations for molecular dynamics simulations. Journal of computational chemistry 2009, 30, 2157-2164.

(55) Hoover, W. G. Canonical dynamics: equilibrium phase-space distributions. Physical review A 1985, 31, 1695.

(56) Goh, G. B.; Eike, D. M.; Murch, B. P.; Brooks III, C. L. Accurate modeling of ionic surfactants at high concentration. The Journal of Physical Chemistry B 2015, 119, $6217-6224$. 
(57) Seaton, M. A. DL_MESO_DPD: development and use of mesoscale modelling software. Molecular Simulation 2018, 1-20.

(58) González-Melchor, M.; Mayoral, E.; Velázquez, M. E.; Alejandre, J. Electrostatic interactions in dissipative particle dynamics using the Ewald sums. The Journal of chemical physics 2006, 125, 224107.

(59) Bray, D. J.; Del Regno, A.; Anderson, R. L. in preparation.

(60) Camesasca, M.; Kaufman, M.; Zloczower, I. M. Quantifying fluid mixing with the Shannon entropy. Macromolecular theory and simulations 2006, 15, 595.

(61) Jiang, H.; Vogtt, K.; Thomas, J. B.; Beaucage, G.; Mulderig, A. Enthalpy and entropy of scission in wormlike micelles. Langmuir 2018, 34, 13956-13964.

(62) Terreau, O.; Bartels, C.; Eisenberg, A. Effect of poly (acrylic acid) block length distribution on polystyrene-b-poly (acrylic acid) block copolymer aggregates in solution. 2. A partial phase diagram. Langmuir 2004, 20, 637-645.

(63) Schmitt, A. L.; Repollet-Pedrosa, M. H.; Mahanthappa, M. K. Polydispersity-driven block copolymer amphiphile self-assembly into prolate-spheroid micelles. ACS Macro Letters 2012, 1, 300-304.

(64) Christov, N.; Denkov, N.; Kralchevsky, P.; Ananthapadmanabhan, K.; Lips, A. Synergistic sphere-to-rod micelle transition in mixed solutions of sodium dodecyl sulfate and cocoamidopropyl betaine. Langmuir 2004, 20, 565-571.

(65) Abdel-Rahem, R. A.; Reger, M.; Hloucha, M.; Hoffmann, H. Rheology of aqueous solutions containing SLES, CAPB, and microemulsion: Influence of cosurfactant and salt. Journal of Dispersion Science and Technology 2014, 35, 64-75. 


\section{Graphical TOC Entry}

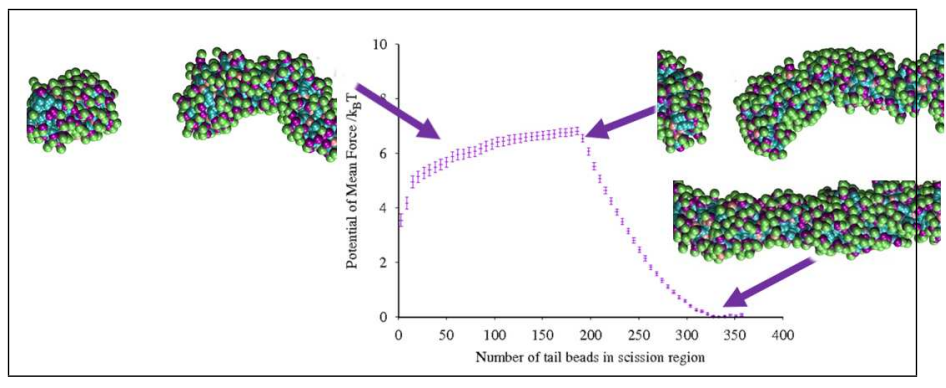

Some journals require a graphical entry for the Table of Contents. This should be laid out "print ready" so that the sizing of the text is correct. Inside the tocentry environment, the font used is Helvetica 8 pt, as required by Journal of the American Chemical Society.

The surrounding frame is $9 \mathrm{~cm}$ by $3.5 \mathrm{~cm}$, which is the maximum permitted for Journal of the American Chemical Society graphical table of content entries. The box will not resize if the content is too big: instead it will overflow the edge of the box.

This box and the associated title will always be printed on a separate page at the end of the document. 